\title{
Efficient Gene Stacking in Rice Using the GAANTRY System
}

\author{
Leyla T. Hathwaik, James Horstman, James G. Thomson and Roger Thilmony * (])
}

\begin{abstract}
Genetic engineering of rice provides a means for improving rice grain quality and yield, and the introduction and expression of multiple genes can produce new traits that would otherwise be difficult to obtain through conventional breeding. GAANTRY (Gene Assembly in Agrobacterium by Nucleic acid Transfer using Recombinase technologY) was previously shown to be a precise and robust system to stably stack ten genes (28 kilobases (kb)) within an Agrobacterium virulence plasmid Transfer-DNA (T-DNA) and obtain high-quality Arabidopsis and potato transgenic events. To determine whether the GAANTRY system can be used to engineer a monocotyledonous crop, two new T-DNA constructs, carrying five (16.9 kb) or eleven ( $37.4 \mathrm{~kb}$ ) cargo sequences were assembled and transformed into rice. Characterization of 53 independent transgenic events demonstrated that more than $50 \%$ of the plants carried all of the desired cargo sequences and exhibited the introduced traits. Additionally, more than $18 \%$ of the lines were high-quality events containing a single copy of the introduced transgenes and were free of sequences from outside of the T-DNA. Therefore, GAANTRY provides a simple, precise and versatile tool for transgene stacking in rice and potentially other cereal grain crops.
\end{abstract}

Keywords: Agrobacterium, Gene stacking, Genetic engineering, Oryza sativa, Site-specific recombinase

\section{Background}

Rice is one of the main staple food crops which feeds about half of the world's population. It is estimated that by 2030 , the world's population is expected to reach 8 billion, and rice production must increase by $25 \%$ to meet the growing demand (Brar and Khush 2013). The exponential growth of omics (genomics, transcriptomic, proteomic and metabolomics) information offers substantial opportunities toward comprehending the mechanisms underlying rice grain quality and yield, furthermore, the integration of knowledge from this data with genetic engineering could potentially help achieve the needed sustainable increase in rice production.

Genetic engineering of rice has introduced several important traits such as herbicide tolerance, biotic/abiotic stress resistance and the improvement of nutritional value, as well as the quality of improvement of rice grains (Bao 2018; Biswal et al. 2017). The transgenic rice

* Correspondence: Roger.Thilmony@usda.gov

United States Department of Agriculture-Agriculture Research Service,

Western Regional Research Center, Crop Improvement and Genetics Research Unit, Albany, CA 94710, USA lines that have been most frequently developed typically express one or a few genes that confer traits of interest; nevertheless, modifying multiple traits simultaneously or introducing complex biosynthetic pathways will typically require the engineering of multiple genes. Therefore, rice biotechnology would significantly benefit from the application of a simple and versatile system for transgene assembly and efficient plant transformation.

Various different approaches have been utilized previously for the assembly of large plant transformation constructs, including the use of commercial cloning systems i.e. multisite Gateway, homing endonucleases, Gibson assembly, type IIS restriction enzymes, homologous recombination in yeast, as well as the Cre site-specific recombinase (Dafny-Yelin and Tzfira 2007; Ma et al. 2011; Weber et al. 2011; Untergasser et al. 2012; Zeevi et al. 2012; Buntru et al. 2013; Binder et al. 2014; Shih et al. 2016; Cermak et al. 2017; Zhang et al. 2017; Zhu et al. 2017). These approaches typically utilize either a binary vector plasmid or a binary bacterial artificial chromosome plasmid vector as the platform for the plant transformation construct. These efforts have produced large and

\section{Springer Open}

(c) The Author(s). 2021 Open Access This article is licensed under a Creative Commons Attribution 4.0 International License, which permits use, sharing, adaptation, distribution and reproduction in any medium or format, as long as you give appropriate credit to the original author(s) and the source, provide a link to the Creative Commons licence, and indicate if changes were made. The images or other third party material in this article are included in the article's Creative Commons licence, unless indicated otherwise in a credit line to the material. If material is not included in the article's Creative Commons licence and your intended use is not permitted by statutory regulation or exceeds the permitted use, you will need to obtain permission directly from the copyright holder. To view a copy of this licence, visit http://creativecommons.org/licenses/by/4.0/. 
complex multi-gene constructs, but in some cases have also been limited by construct instability problems in bacteria. Additionally, some were only shown to function in transient expression assays or in model plants, and/or were not able to efficiently generate stable transgenic plants exhibiting all the desired functional phenotypes. Site-specific recombinases have also been used to sequentially stack transgenes within a soybean target locus, and more recently within the rice genome ( $\mathrm{Li}$ et al. 2010; Nandy et al. 2015; Pathak and Srivastava 2020), but these approaches require the generation of target lines with introduced sequences and perform stacking with multiple rounds of plant transformation. Clearly, plant biotechnology would significantly benefit from the application of a simple and versatile system that allows for the easy and stable assembly of large stacked constructs and their subsequent efficient and high-fidelity introduction into crop plants (Srivastava and Thomson 2016).

The GAANTRY (Gene Assembly in Agrobacterium by Nucleic acid Transfer using Recombinase technology) system has proven to be an efficient method of gene assembly and plant transformation that generates high-quality transgenic Arabidopsis and potato plants (Collier et al. 2018; McCue et al. 2019). The GAANTRY system is a simple to use, yet sophisticated method that allows the in vivo stacking of multiple transgenes within an Agrobacterium virulence plasmid Transfer-DNA (T-DNA). This system takes advantage of three unidirectional recombinases that mediate site-specific DNA recombination at their specific DNA recognition sites. The TP901 and A118 recombinases perform integration by recognition of their specific attB and attP recognition sites, while the ParA recombinase performs excision through its recognition of two directly oriented MRS recognition sites (Keravala et al. 2006; Thomson and Ow 2006; Thomson et al. 2009). The assembly of cargo sequences within the GAANTRY's T-DNA is an efficient, simple and modular process that only requires three types of components; 1 ) the modified GAANTRY recipient Agrobacterium strain, 2) two cloning plasmids (B and P Donors) for the insertion of sequence(s) of interest and 3) two accessory plasmids ( $B$ and $P$ Helpers) that carry an operon expressing two of the recombinases (for a detailed description of how the GAANTRY assembly process functions, see Collier et al. 2018). Recently, the GAANTRY system was demonstrated to efficiently assemble a 28.5 kilobase $(\mathrm{kb})$ 10-stack T-DNA and produce high-quality genetically engineered Arabidopsis and potato plants (Collier et al. 2018; McCue et al. 2019).

To further investigate the use of GAANTRY as a tool to genetically modify monocotyledonous crop plants, we sequentially stacked 5 or 11 cargo sequences to generate a 16.9 or $37.4 \mathrm{~kb}$ T-DNA respectively and produced genetically engineered rice. Transgenic events were evaluated phenotypically and genotypically to determine the completeness of T-DNA transfer and integration, as well as the quality of the events based of copy number and presence of sequences outside of the T-DNA's left border (LB).

\section{Materials and Methods \\ Construction of B and P Donors}

Genes of interest (promoter/gene coding sequence/terminator) were assembled into either a B or P donor plasmid (Collier et al. 2018) using conventional restrictionligation cloning methods. Ligated $\mathrm{P} / \mathrm{B}$ donors were transformed and maintained in E.coli $\mathrm{DH} 5 \alpha$ and plasmid DNA was extracted using a plasmid isolation kit according to manufacturer's instructions (Zymo Research, CA). A summary of the $\mathrm{P} / \mathrm{B}$ donors and their cargo sequences used to assemble the 5-stack and 11-stack T-DNAs is shown in Table 1. These plasmids and their complete annotated sequences are available on request.

\section{Multigene Stacking in the GAANTRY Strain}

The GAANTRY transgene stacking method (Collier et al. 2018) was used as previously described to stack cargo sequences in five or eleven steps within the GAANTRY ArPORT1 strain (Fig. 1 and 2a). Briefly, GAANTRY assembly is a simple process that employs iterative recombinase-mediated integration and excision reactions that toggle between the $\mathrm{B}$ Donor vectors (conferring bacterial gentamicin resistance) and $\mathrm{P}$ Donor vectors (conferring bacterial kanamycin resistance) each carrying the desired cargo. The $\mathrm{B}$ and $\mathrm{P}$ Donor vectors that were used to generate 5- and 11stack GAANTRY strains are summarized in Table 1. Note that the nptII expression cassette in the 11stack construct was strategically added as the last stack to avoid any potential problems that would result from making the GAANTRY strain constitutively resistant to kanamycin (if the ZmUbilp-nptII expression cassette expressed in bacteria), which would make adding additional cargoes difficult with P Donor vectors carrying cargo sequences. The molecular characterization and validation of the strains generated from each stacking event involved picking three random colonies and performing genomic PCR reactions using gene specific primers that span the junctions between preexisting sequences and newly inserted cargo. The PCR amplification conditions used were an initial denaturation step of $5 \mathrm{~min}$ at $95^{\circ} \mathrm{C}$, followed by 35 cycles of $30 \mathrm{~s}$ at $95^{\circ} \mathrm{C}, 30 \mathrm{~s}$ at $56^{\circ} \mathrm{C}$ and $2 \mathrm{~min}$ at $68^{\circ} \mathrm{C}$, with a final extension at $68^{\circ} \mathrm{C}$ for $5 \mathrm{~min}$. The primers used for this analysis are shown in Supplemental Table 1. 
Table 1 List of B and P Donor vectors used to assemble the 5-stack and 11-stack T-DNA

\begin{tabular}{|c|c|c|c|c|c|c|}
\hline \multirow{2}{*}{$\begin{array}{l}\text { Stack } \\
\#\end{array}$} & \multirow{2}{*}{$\begin{array}{l}\text { Donor } \\
\text { Vector }\end{array}$} & \multirow[b]{2}{*}{ Cargo Size (kb) } & \multirow[t]{2}{*}{ Phenotype } & \multicolumn{3}{|c|}{ Cargo Sequence } \\
\hline & & & & Promoter & $\mathrm{CDS}^{\mathrm{a}}$ & Terminator \\
\hline 1 & B & 3.4 & hygromycin resistance & $R \cup B Q 2$ & hptll & CaMV 355 \\
\hline 2 & $\mathrm{P}$ & 2.1 & Renilla luciferase activity & CaMV 355 & Rluc & CaMV 355 \\
\hline 3 & $\mathrm{~B}$ & 2.2 & enhancer blocking insulator & \multicolumn{3}{|c|}{ TBS (Transformation Booster Sequence) } \\
\hline 4 & $P$ & 4.2 & firefly luciferase activity & $\mathrm{OsCcl}$ & Fluc & nos \\
\hline 5 & $\mathrm{~B}$ & 4.8 & $\beta$-glucuronidase activity & OsLP2 & GUSPlus & nos \\
\hline 6 & $\mathrm{P}$ & 3.1 & glufosinate herbicide tolerance & PvUbil & bar & nos \\
\hline 7 & B & 3.0 & green florescence & OsRoot6 & eGFP & CaMV 355 \\
\hline 8 & $\mathrm{P}$ & 2.2 & enhancer blocking insulator & \multicolumn{3}{|c|}{ TBS (Transformation Booster Sequence) } \\
\hline 9 & B & 4.3 & red florescence ${ }^{b}$ & OsPS2 & tdTomato $^{E R}$ & nos $/ 355$ \\
\hline 10 & $P$ & 4.6 & glyphosate herbicide tolerance & OsGOS2 & EPSPS & nos \\
\hline 11 & $\mathrm{~B}$ & 3.4 & paromomycin resistance & ZmUbil & nptll & CaMV 355 \\
\hline
\end{tabular}

${ }^{a}$ CDS: gene coding sequence

${ }^{\mathrm{b}}$ The cargo 9 sequence was nonfunctional and failed to produce detectable red fluorescent pollen

Examining the Stability of the GAANTRY Stacked Cargo in Agrobacterium

To determine the stability of the stacked cargo within the GAANTRY strain, the 5-stack and 11- stack strains were subcultured every $24 \mathrm{~h}$ over a period of 6 days in nonselective LB broth media and subsequently plated on nonselective LB agar. One hundred colonies were then picked and restreaked on LB media containing gentamicin. Then 10 colonies were randomly selected for cargo validation PCR using the primers shown in Supplemental Table 1.

\section{Generation of 5-Stack and 11-Stack Rice Plants}

To generate transgenic rice (Oryza sativa) cv. Nipponbare plants, seed-derived embryogenic calli were cocultured with Agrobacterium rhizogenes GAANTRY strain carrying either the 5-stack or 11-stack T-DNA as previously described (Cook and Thilmony 2012). A single Agrobacterium -rice callus co-cultivation experiment was performed using the 5-stack strain to recover 16 independent events, while two separate co-cultivation experiments were performed with the GAANTRY 11-stack strain to generate 37 independent events. Hygromycin antibiotic selection $(40 \mathrm{mg} / \mathrm{L})$ was used to identify transgenic rice callus. Regenerated shoots were grown in the greenhouse at $28^{\circ} \mathrm{C}$ with $16 \mathrm{~h}$ of light in Sunshine Mix \#1 soil medium (Sun Gro Horticulture, WA).

\section{Phenotypic Analysis of $\mathrm{T}_{1}$ Transgenic Rice Plants Antibiotic and Herbicide Resistance}

To determine antibiotic and herbicide resistance of GAANTRY transgenic rice lines, selective germination

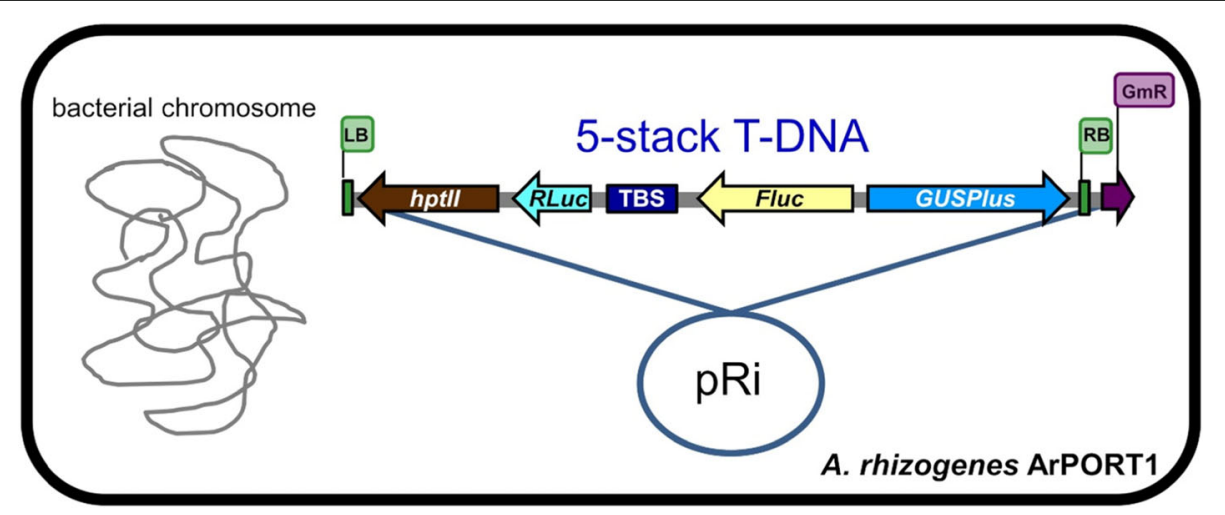

Fig. 1 Composition of the 5-stack T-DNA. Diagram of an Agrobacterium rhizogenes virulence plasmid containing the 5-stack T-DNA. Cargo sequences, including the promoter, gene coding sequence and transcription terminator, are displayed as a colored arrow showing the orientation of transcription or a dark blue rectangle (TBS insulator). The T-DNA right border (RB) and left border (LB) locations are shown in green. Abbreviations are as follows: hptll, hygromycin phosphotransferase 2, Rluc (Renilla luciferase), TBS: Transformation Booster Sequence, Fluc (Firefly luciferase) and GUSPlus (B-glucuronidase encoding reporter gene). The gentamicin bacterial resistance marker $(G m R)$ is shown outside the RB region of the T-DNA, within the native Agrobacterium virulence plasmid (pRi) 


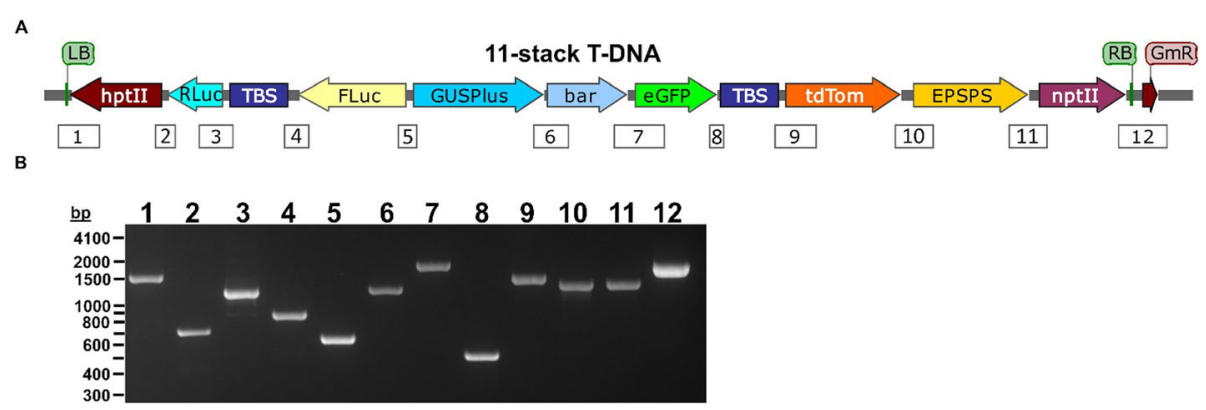

Fig. 2 Composition of the 11-stack T -DNA and PCR validation of cargo sequences. a Diagram of the GAANTRY 11-stack 37.4 kb T-DNA. See Fig. 1 legend for a description of the diagram. PCR products that bridge junctions between each cargo sequence are indicated by the numbered rectangles below the T-DNA. $\mathbf{b}$ Gel electrophoresis image of the PCR amplicons spanning each junction. Abbreviations are as follows: $h p t l l$ (hygromycin phosphotransferase 2), Rluc (Renilla luciferase), TBS (Transformation Booster Sequence), Fluc (Firefly luciferase), GUSPlus (ß-glucuronidase encoding reporter gene), bar (bialaphos resistance), eGFP (enhanced Green Fluorescent Protein), tdTom (tdTomato red fluorescent protein), EPSPS (5enol-pyruvylshikimate-3-phospate synthase), and nptll (neomycin phosphotransferase 2)

and herbicide application assays were performed. Briefly, seeds were harvested from the $\mathrm{T}_{0}$ plants, dehulled, surface sterilized and subsequently transferred into $1 \times \mathrm{MS}$ medium containing the antibiotic and/or herbicide. The chemicals used were hygromycin $(40 \mathrm{mg} / \mathrm{L})$, paromomycin $(125 \mathrm{mg} / \mathrm{L})$, Finale ${ }^{\circ}$ herbicide $(22.5 \mu \mathrm{l} / \mathrm{L}$, with a final concentration of $2.71 \mathrm{mg} / \mathrm{L}$ glufosinate), and Roundup ${ }^{\circ}$ herbicide $(12.2 \mu \mathrm{l} / \mathrm{L}$, with a final concentration of 4.3 $\mathrm{mg} / \mathrm{L}$ glyphosate) for seedling selection. The seeds on the selective media were placed in a growth chamber (Conviron, CA, USA) under $16 \mathrm{~h}$ of light at $28^{\circ} \mathrm{C}$. The seed germination and seedling growth was evaluated after 1 week. Hygromycin resistant seedlings were transferred to soil, grown in the greenhouse, and used to for scoring the other introduced phenotypes. In addition, Finale ${ }^{\bullet}$ herbicide (1:128 dilution, with a final concentration of $0.93 \mathrm{~g} / \mathrm{L}$ glufosinate) was painted onto three-inch sections of leaves of one-month old plants with a herbicide dipped paint brush and scored for tolerance 7 days post application.

\section{Dual Luciferase Reporter Assay}

Renilla and firefly luciferase activities were measured in a single sample using two leaf disks $(1 \mathrm{~cm}$ in diameter) using the dual-luciferase ${ }^{\circ}$ reporter assay according to manufacturer's instructions (Promega-E1910, WI, USA).

\section{Microscopic Analysis}

A Leica Microsystems-MZ16F stereomicroscope (Bannockburn, IL, USA) was used to examine green fluorescence in one-week-old roots and red fluorescence in pollen. The eGFP fluorescence visualization used an excitation wavelength of $488 \mathrm{~nm}$ with an emission filter 510-530 nm; for tdTomato fluorescence, an excitation wavelength of $558 \mathrm{~nm}$ with an emission filter 583-592 $\mathrm{nm}$ was used.

\section{Histochemical GUSPlus Staining}

To assay $\beta$-glucuronidase reporter enzyme activity, oneweek old seedling leaves or whole seedlings were incubated with histochemical staining solution $(0.1 \mathrm{M}$ sodium phosphate $\mathrm{pH} 7.0,0.5 \mathrm{mM}$ potassium ferrocyanide, $1.5 \mathrm{~g} / \mathrm{L}$ X-gluc (5-bromo-4-chloro-3-indolyl- $\beta$-Dglucuronic acid), and $0.5 \% v / v$ Triton $\mathrm{X}-10$ ) for $18 \mathrm{~h}$ at $37^{\circ} \mathrm{C}$ (Jefferson et al. 1987).

\section{Genotypic Analysis of $T_{1}$ Transgenic Plants}

Genomic rice DNA was extracted using the PureGene tissue DNA isolation kit (Qiagen, Valencia, CA, USA) from leaf tissue samples. Transgenic plants were analyzed by PCR using the same sequence-specific primers and amplification conditions that were used in the 5stack and 11-stack GAANTRY strain validation, as well as internal primers that anneal within individual cargo sequences (those marked with an asterisk, see Figs. 4 and 5) to verify the presence or absence of the cargo sequences (Supplemental Table 1).

\section{Measurement of Transgene Copy Number}

The copy number of the hptII, GUSPlus and nptII cargo sequences were measured using droplet digital PCR (ddPCR) as previously described (Collier et al. 2017). Briefly, rice genomic DNA was isolated (Lassner et al. 1989) and quantified using a dsDNA quantification kit and a Qubit fluorometer according to the manufacturer's instructions (Thermo Fisher Scientific, MA, USA). DNA was digested using an overnight incubation at $37^{\circ} \mathrm{C}$ with the NcoI restriction enzyme (New England BioLabs, MA, USA). A total of $20 \mathrm{ng}$ of digested DNA was used for each ddPCR reaction. Rice OsUBC (Ubiquitin-Conjugating Enzyme E2; LOC_Os02g42314) (Jain et al. 2006) gene was used the single copy reference gene. The reference gene probe was 5' FAM (6-fluorescein)-labeled, and the transgene probes were $5^{\prime}$ HEX (hexachloro- 
fluorescein)-labeled. Primers and probes used for ddPCR analyses are shown in Supplemental Table 4.

\section{Screening for 'Backbone' Sequences from outside the T- DNA Left Border}

Transgenic rice plants were analyzed for the presence of vector backbone sequence beyond the T-DNA left border using genomic PCR. The "LB" primers were designed to amplify a $1142 \mathrm{bp}$ fragment that included sequences outside of the left border repeat ( $230 \mathrm{bp}$ ) and the 3 ' end coding sequence of the hptII gene (755 bp) (within the TDNA). Internal primers (PCR reaction $1^{*}$ ) which amplify a 435 bp fragment within the $3^{\prime}$ end coding sequence of the hptII gene were used as a positive control for the presence of cargo 1 and to be certain that negative PCR results were not caused by insufficient template or a faulty PCR amplification reaction. Additionally, the GAANTRY strain carrying the 11-stack T-DNA was also used as a positive control for backbone sequence. See Supplemental Table 1 for primer sequences.

\section{Results}

Assembly of the 5 and 11-Stack GAANTRY Strains

The GAANTRY transgene stacking method was used to sequentially stack five or eleven sequences within the Agrobacterium virulence plasmid T-DNA (Fig. 1 and 2a). The selected cargo sequences (Table 1) were cloned into a P or B Donor plasmids, and multigene stacking within the GAANTRY ArPORT1 strain was achieved by alternating the use of $\mathrm{B}$ and $\mathrm{P}$ Donor vectors and gentamicin and kanamycin selection to stack the genes of interest into the recipient GAANTRY strain as previously described (Collier et al. 2018). The donors contained transcriptional units that were designed to confer functional phenotypes (i.e. antibiotic/herbicide resistance or reporter gene activity) and one cargo (which appears twice in the 11-stack TDNA) was an enhancer-blocking insulator. The 5-stack GAANTRY strain contains the hptII, Renilla luciferase, TBS insulator, firefly luciferase and GUSPlus cargoes, while the bar, eGFP, TBS insulator, tdTomato, EPSPS and nptII cargo sequences were added to the 5-stack to generate the 11-stack GAANTRY strain (Table 1).

The Donors carrying each cargo sequence were iteratively stacked in either five or eleven independent steps to produce the 5 -stack $(16.9 \mathrm{~kb})$ and 11 -stack $(37.4 \mathrm{~kb})$ T-DNAs respectively (Fig. 1 and 2a). Although the 5and 11-stack T-DNAs could have been constructed in fewer steps by designing donors that contained multiple cargos using Golden Gate-compatible and Gatewaycompatible auxiliary donor vectors (Collier et al. 2018), the 5 and 11 step pathways were chosen to demonstrate the repetitive and efficient nature of the GAANTRY assembly system. Thorough molecular characterization of the assemblies was performed on three randomly selected Agrobacterium colonies via genomic PCR using gene-specific primers that span each of the recombination junctions between the cargo sequences (Fig. 2). All of the junctions in all tested colonies during each round of assembly were validated by genomic PCR screening, demonstrating the efficiency and reliability of the GAANTRY-mediated cargo insertion process. Approximately equal number of colonies were obtained during each round of transformation from stack 1 to stack 11 .

\section{Stability of the Stacked Cargo Sequences}

To determine the stability of the stacked cargo within the ArPORT1 GAANTRY strain pRi plasmid, the 5stack and 11-stack strains were subcultured in nonselective LB broth medium every $24 \mathrm{~h}$ over a period of 6 days. Aliquots of the final culture were then plated on nonselective LB agar, and one hundred random colonies were screened using gentamicin selection. All one hundred colonies exhibited antibiotic resistance; ten of these were randomly selected for validation with genomic PCR and were confirmed to carry all expected cargo sequences. Representative PCR results are shown in Fig. $2 b$ for an individual 11-stack clone.

\section{Generation and Characterization of 5-Stack and 11-Stack Transgenic Rice Plants}

To examine the ability of the GAANTRY strains to generate transgenic rice, Nipponbare wild-type embryogenic rice callus was co-cultured with either the 5 or 11-stack GAANTRY strains. Transgenic calli was identified using hygromycin antibiotic selection. Sixteen and thirty-seven independent transgenic $\left(\mathrm{T}_{0}\right)$ events were recovered using the GAANTRY 5- and 11-stack strains respectively. The $\mathrm{T}_{0}, \mathrm{~T}_{1}$ and $\mathrm{T}_{2}$ generations of each event were grown in the greenhouse to determine the functionality and stability of the introduced T-DNAs. Phenotypic and genotypic results indicate stable inheritance of the transgenes in each of the transgenic lines with no obvious differences observed in morphology or growth between the genetically engineered and wild-type Nipponbare rice plants.

The phenotypic expression of the introduced traits was analyzed in for each of the transgenic events. Cargo sequences 1 and 11 (Table 1) confer antibiotic resistance. The hptII gene is under the control of the rice $u b i$ quitin 2 promoter and nptII expression is conferred by the maize ubiquitin 1 promoter; these genes confer resistance to hygromycin and paromomycin respectively (Collier et al. 2016). Germination assays were performed for $\mathrm{T}_{1}$ seed using antibiotic selection. As expected, all transgenic events produced progeny that were resistant to hygromycin, consistent with that antibiotic being used to select the original $T_{0}$ transgenic events. A representative example of the hygromycin resistance observed is shown in Fig. 3a. The $\mathrm{T}_{1}$ seed from all 37 of the 11- 


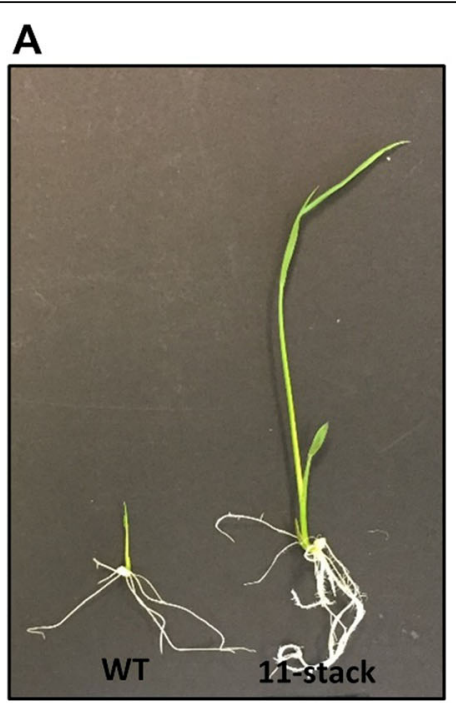

C

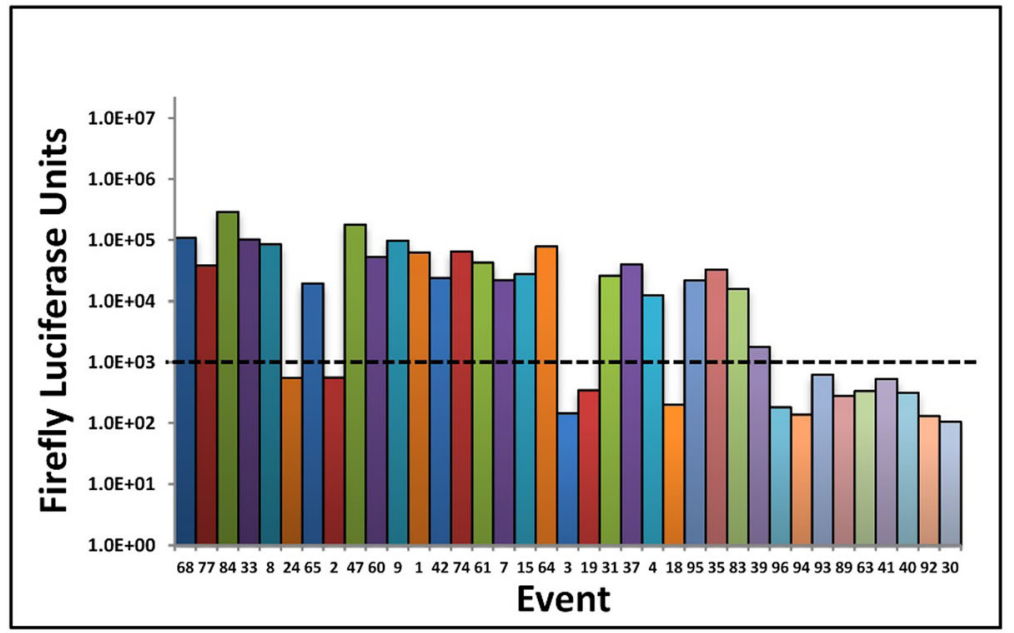

E

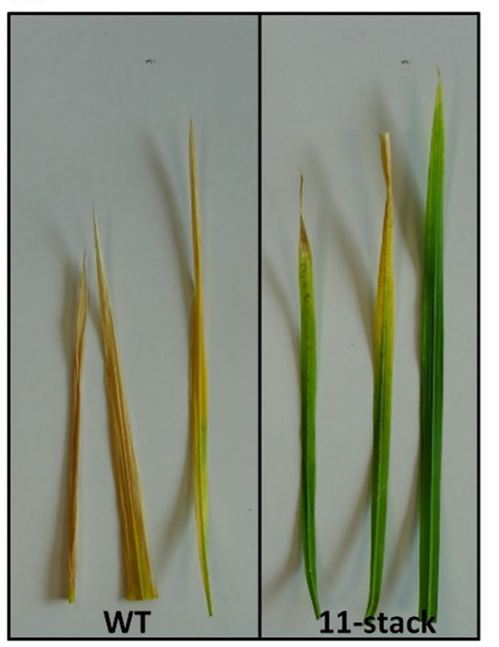

B

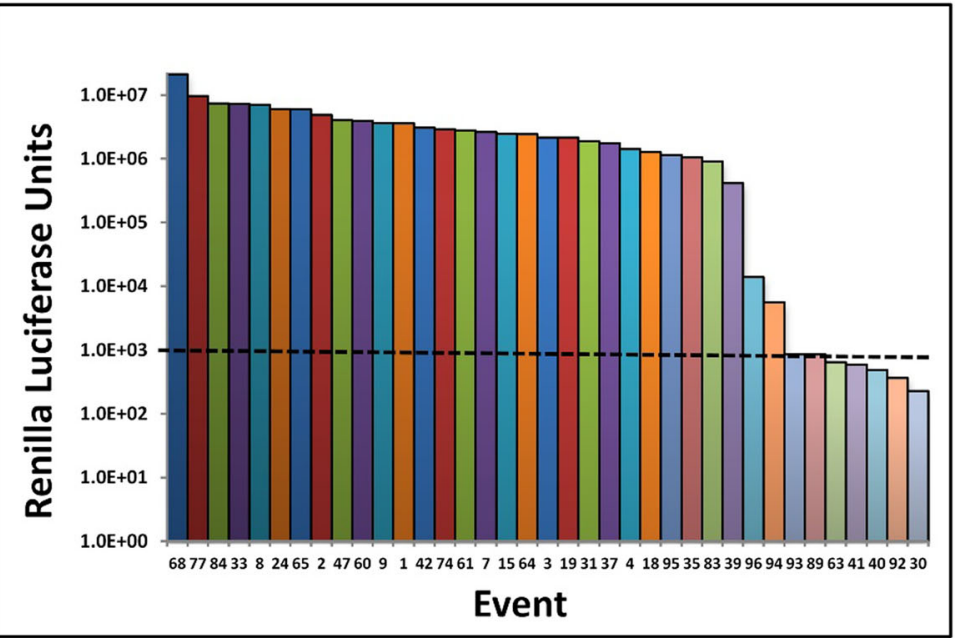

D

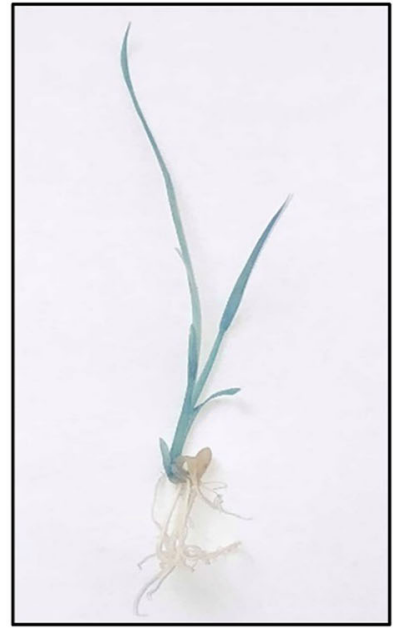

$F$

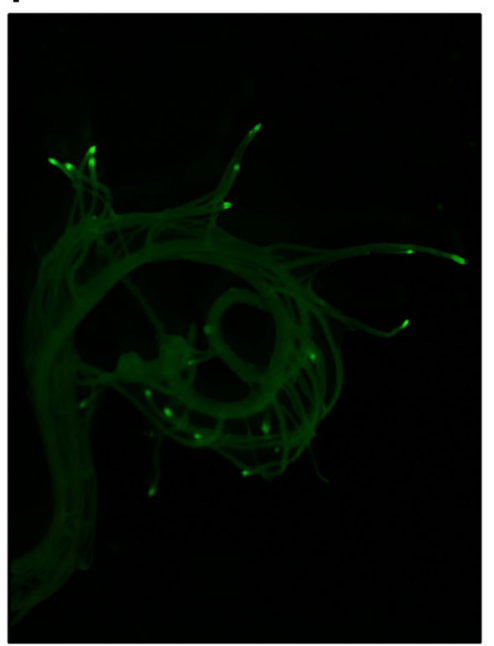

G

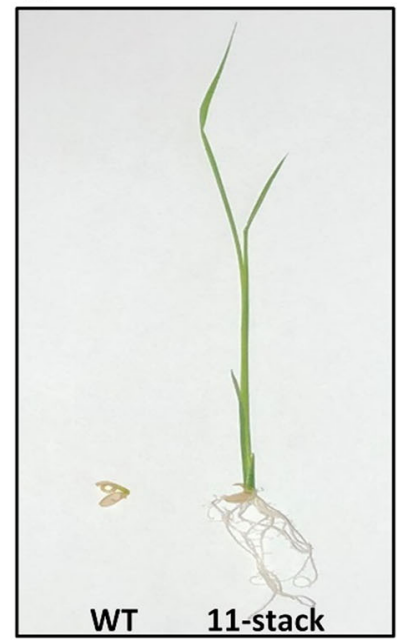

Fig. 3 (See legend on next page.) 
(See figure on previous page.)

Fig. 3 Representative phenotypes observed in the GAANTRY 11-stack T1 rice plants. a Hygromycin sensitive wildtype Nipponbare (WT, left) and resistant (11-stack right) seedlings 7 days post germination. b Measured Renilla luciferase activity for 11-stack leaf samples. c Firefly luciferase activity for 11-stack leaf samples. The average background levels of activity detectable in wildtype leaf extracts is shown with the dashed line at $1.0 \times 10^{3}$ units. $\mathbf{d} \beta$-glucuronidase activity detected in green tissues and not in roots in a histochemically stained 7-day old 11-stack seedling. e Finale herbicide sensitivity in wildtype (left) and tolerance in 11-stack (right) leaves. $\mathbf{f}$ Observed green fluorescence localized in the root tips of a 11-stack rice plant. $\mathbf{g}$ The phenotypes of a wildtype (left) and 11-stack (right) seedlings germinated on media containing hygromycin and paromomycin antibiotics as well as glufosinate and glyphosate herbicides

stack events were germinated on paromomycin media and $73 \%$ had progeny that exhibited resistance (Supplemental Table 3; Fig. 3g). Cargo sequences 2 and 4 were expected to confer Renilla and firefly luciferase activity (Table 1). A dual-luciferase activity assay was performed on leaf tissue extracts to assess which events had functional expression for each reporter gene. All 5-stack events exhibited Renilla luciferase activity and $81 \%$ had detectable firefly luciferase activity. Genomic PCR on the three events lacking activity confirmed that those plants also lacked at least a portion of the firefly luciferase expression cassette (Fig. 4, Supplemental Table 2). For the 11-stack events, $81 \%$ had detectable Renilla luciferase activity and $62 \%$ were positive for firefly luciferase activity (Fig. 3b-c, Supplemental Table 3). Similar to the 5stack transgenic events, all of the 11-stack events that did not have luciferase activity also lacked that portion of the 11 stack T-DNA (Fig. 5, Supplemental Table 3).

Cargo sequences 3 and 8 were the Transformation Booster Sequence (TBS) from Petunia (Hily et al. 2009). This sequence is expected to block interactions between the CaMV 35S enhancer and nearby transgenes within the T-DNAs, thus maintaining the fidelity of their organ- or tissue-specific expression. The fifth cargo sequence was the GUSPlus expression cassette (Table 1) where expression of the reporter was controlled by the rice organ-specific Leaf Panicle 2 promoter (Thilmony et al. 2009). For the 5 -stack events, $56 \%$ exhibited the expected green tissue-specific $\beta$-glucuronidase activity via histochemical staining (Supplemental Table 2). Approximately $54 \%$ of the 11 -stack events had detectable GUSPlus reporter activity only in leaves and other photosynthetic tissues (Fig. 3d, Supplemental Table 3). Most of the events lacking activity also lacked the GUSPlus portion of the T-DNA based on genomic PCR and ddPCR screening, although two of the 5-stack events and two of the 11-stack events carried at least a part of the GUSPlus construct sequence but failed to produce detectable histochemical staining (Figs. 4, 5, Supplemental Tables 2, 3).

The sixth cargo sequence in the 11-stack T-DNA was the bar gene under control of the constitutive switchgrass Ubiquitin 1 promoter (PvUbi1) (Mann et al. 2011). Finale $^{\bullet}$ herbicide was applied to individual leaves and

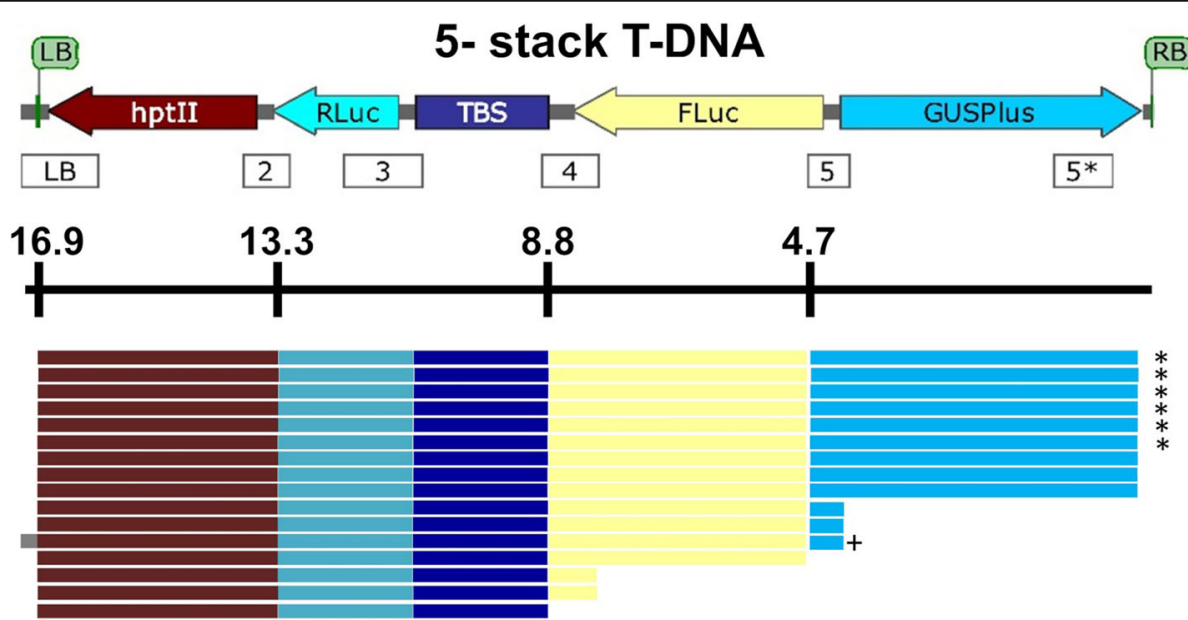

Fig. 4 The GAANTRY 5-stack T-DNA and summary of the phenotypes and genotypes of the independent events. The structure of the $16.9 \mathrm{~kb} 5$ stack GAANTRY T-DNA (top). PCR amplicons used to determine the presence of the T-DNA cargo sequences and the LB backbone sequence are indicated by the numbered rectangles below the T-DNA diagram. Amplicon $5^{*}$ is fully within the GUSPlus cargo sequence, while the other amplicons span the junction between cargo sequences. The distance of each complete cargo sequence is from the right border (in kb) is shown below the diagram. Colored rectangles indicate the presence of the associated phenotype and/or genotype for each independent transgenic event. The asterisks $\left(^{*}\right)$ mark the events with a single copy of the hptll and GUSPlus transgenes. The plus sign $(+)$ marks the event that contained sequence outside of the T-DNA beyond the left border 


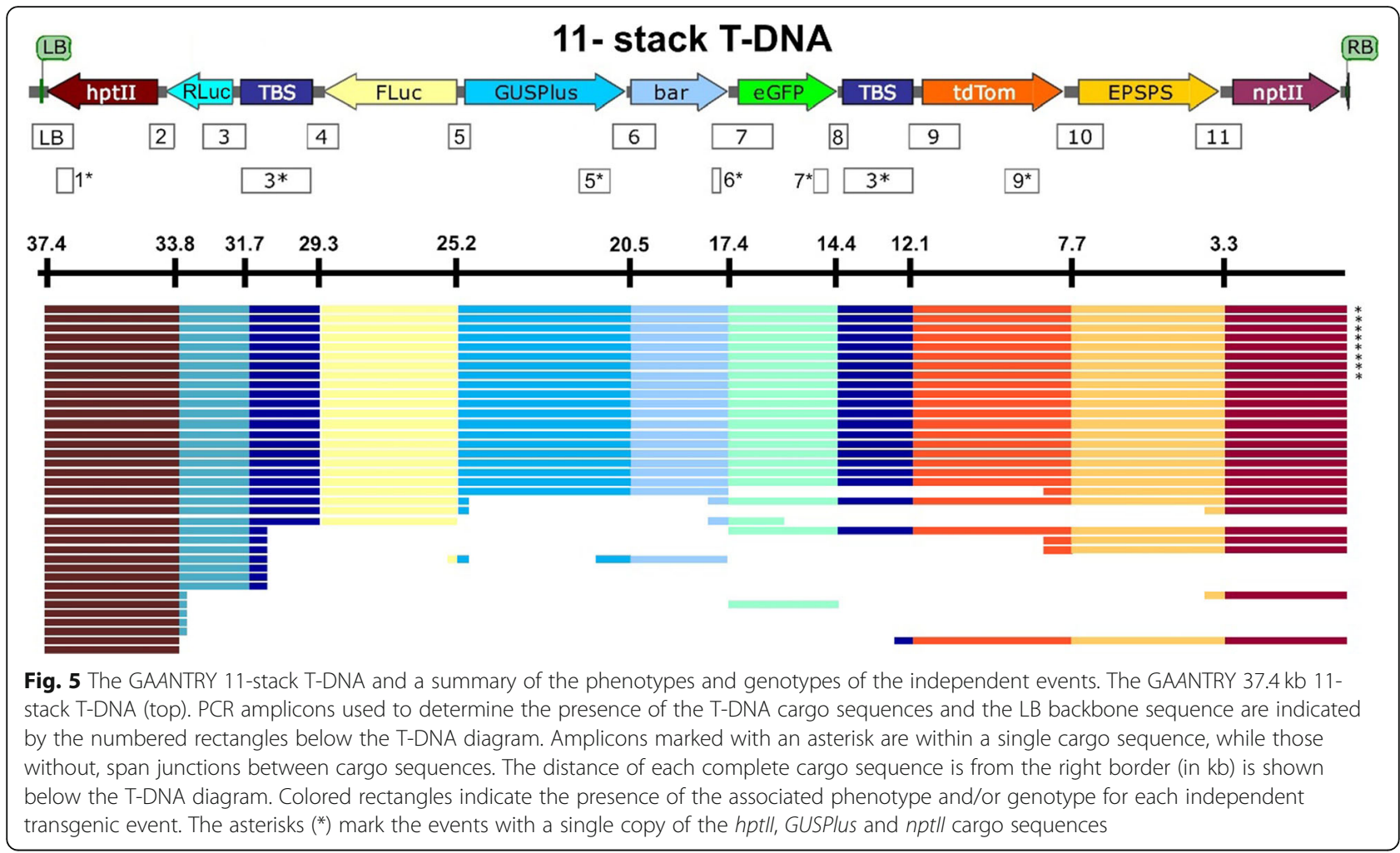

$57 \%$ of the events showed tolerance (Fig. 3e). The events that were as sensitive to Finale ${ }^{\bullet}$ herbicide as the wildtype Nipponbare plants lacked the region of the T-DNA with the bar cargo sequence (Fig. 5, Supplemental Table 3). The seventh cargo sequence contained the eGFP (enhanced Green Fluorescence Protein) gene with its expression controlled by the rice Root 6 promoter which controls root-tip specific expression (Xing et al., unpublished). A total of 22 of the events (60\%) exhibited root-tip specific green fluorescence and carried that portion of the 11stack T-DNA (Fig. 3f, Supplemental Table 3). Cargo sequence 9 carried the red fluorescent tdTomato reporter gene under control of the rice PS2 (also known as OsLPS3) pollen-specific promoter sequence (Oo et al. 2014). Even though $60 \%$ of the transgenic events carried the complete $t d$ Tomato expression cassette based on genomic PCR, none of the events exhibited detectable red fluorescence in pollen, or any other tissues (Fig. 5, Supplemental Table 3). Since the tdTomato transgene failed to generate a detectable phenotype in any of the 11-stack events, this sequence was considered a nonfunctional cargo sequence. The tenth cargo sequence was the 5-enolpyruvylshikimate-3-phospate synthase (EPSPS) gene under the control of the rice GOS2 promoter (de Pater et al. 1992). Seedling germination assays determined that $68 \%$ of the events were tolerant to Round-up herbicide and genomic PCR screening confirmed that the 12 events that lacked herbicide tolerance, also lacked the EPSPS portion of the 11-stack T-DNA (Supplemental Table 3). Similarly, $\mathrm{T}_{1}$ seeds from each of the 11-stack events was germinated on paromomycin and $73 \%$ of the events exhibited antibiotic resistance and carried the 11th cargo sequence (Supplemental Table 3). Additionally, to confirm that the 11-stack plants can be both antibiotic and herbicide tolerant, a germination assay was performed with media that simultaneously contained hygromycin, paromomycin, glufosinate and glyphosate. An image of a sensitive wildtype Nipponbare seedling and a resistant/tolerant 11-stack seedling is shown in Fig. 3g.

Overall, for the GAANTRY 5-stack events, 56\% contained all four expected phenotypes (Fig. 4). Of the remaining events, $43 \%$ were lacking $\beta$-glucuronidase and $13 \%$ were missing firefly luciferase and $\beta$-glucuronidase activity. In the case of the GAANTRY 11-stack events, $51 \%$ contained all eight functional phenotypes and appeared to have at least one copy of the entire $37 \mathrm{~kb}$ TDNA. The remaining $49 \%$ of the events were missing one or more of the functional phenotypes and two or more of the cargo sequences (Supplemental Table 3). The phenotypes near the center of the T-DNA were the most frequently lacking in this set of events. The observed phenotypic and genotypic results from the 5- and 11-stack events remained stable in the $\mathrm{T}_{2}$ generation, indicating stable inheritance and expression of the integrated transgenes in each event. 


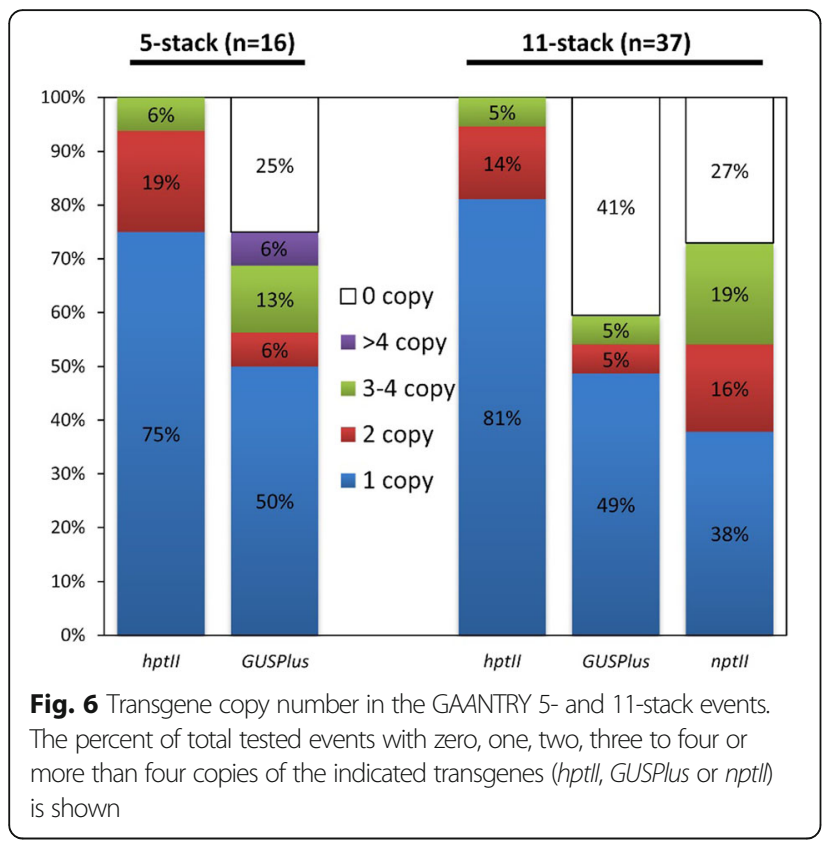

\section{Quantification of Transgene Copy Number and Detection} of Sequences from outside the T-DNA

The transgene copy number was measured using droplet digital PCR (ddPCR) in $\mathrm{T}_{0}$ transgenic 5-stack and 11stack events. A summary of the results is shown in Fig. 6. For the 5 -stack events, $38 \%$ were shown to likely have one copy of a complete T-DNA (i.e. they had 1 copy of both the hptII and GUSPlus transgenes and exhibited all four of the expected phenotypes) (Supplemental Table 2). For the GAANTRY 11-stack events, 19\% were highquality/desirable lines carrying one copy of the hptII, GUSPlus and nptII cargo sequences, and exhibited all eight of the functional phenotypes (Figs. 3 and 5, Supplemental Table 3).

Overall, the copy number for the hptII transgene was quite low, with $75 \%$ and $81 \%$ of the 5 -stack and 11 -stack events having a single copy, respectively (Fig. 6, Supplemental Tables 2, 3). However, only $38 \%$ of the 11 -stack events had a single copy of the nptII cargo sequence. Furthermore, ddPCR results confirmed the lack of portions of the T-DNA that correlated with the missing phenotypes. The transgenic 5-stack and 11-stack GAANTRY rice plants were also screened by genomic PCR for the presence of introduced 'backbone' sequences. One 5stack event (transgenic event 7; Fig. 4, Supplemental Table 2) had DNA from outside T-DNA left border, while the remaining 52 events were free of backbone sequences (Supplemental Tables 2 and 3).

\section{Discussion}

Rapid progress in biotechnology and omics data availability has made it possible to enhance agronomic traits through genetic engineering. In contrast to monogenic traits to engineer herbicide or pest resistance, the genetic engineering of multiple traits or complex traits is more challenging and will involve multiple genes or whole biosynthetic or signaling pathways. Recently, the GAANTRY system was demonstrated to be an effective technology that allowed the in vivo assembly and stable maintenance of a 10-stack $28.5 \mathrm{~kb}$ T-DNA within an Agrobacterium virulence plasmid and to successfully produce high quality transgenic events in both Arabidopsis thaliana and potato plants (Collier et al. 2018; McCue et al. 2019). In this report, the GAANTRY system was tested to assess its capacity to generate transgenic rice plants using new 5-stack and 11-stack assemblies carrying cargo sequences which are more suitable for functioning in monocots. The GAANTRY system was again shown to effectively assemble and stably maintain large multigene T-DNA stacks, as the 11-stack T-DNA was $30 \%$ larger than the previously published 10 -stack, totaling $37.4 \mathrm{~kb}$. The assembly process was also demonstrated to be a simple and reliable process that can allow numerous iterative rounds of the integration of cargo into a GAANTRY Agrobacterium strain TDNA. It was also shown that not only is the assembly process reliable, but the assembled cargo is stably maintained in the GAANTRY strain, even when grown for days without antibiotic selection. Although individual transgene cassettes were assembled into the T-DNA in as many as eleven rounds of site-specific recombination and selection, it is entirely possible that through the use of Golden Gate-compatible and Gateway ${ }^{\circ}$-compatible auxiliary donor vectors (Collier et al. 2018), large multigene assemblies of ten genes or more could be constructed in as few as two or three rounds of GAANTRY assembly, making this a very rapid and efficient process.

The use of the 5-stack and 11-stack GAANTRY strains allowed us to generate genetically engineered rice expressing multiple traits. The utilization of both constitutive and organ-specific promoters to control the expression of the introduced transgenes, in combination with the TBS enhancer-blocking insulator, demonstrated that continuous and spatio-temporal/organ-specific expression could be achieved with a single large T-DNA construct. The 11-stack T-DNA contained constitutively expressed genes for hygromycin and paromomycin resistance, glufosinate and glyphosate herbicide tolerance, and two forms of luminescence conferred by the Renilla and firefly luciferases. In addition, the engineered lines had green tissue organ-specific $\beta$-glucuronidase activity and exhibited detectable green fluorescence only within the root tips. Surprisingly, use of a rice pollen specific promoter fused to the tdTomato reporter gene failed to generate detectable fluorescence in pollen (as expected) or any other tissues. An explanation for this unexpected result is not obvious, since the entire expression cassette was sequence confirmed within the 11-stack T-DNA 
and the tdTomato transgene and PS2 pollen-specific promoter has been confirmed functional in Nipponbare rice in other transformation constructs (data not shown).

Analysis of the 5-stack and 11-stack transgenic plant populations $\left(T_{0}, T_{1}\right.$ and $\left.T_{2}\right)$ that were recovered using hygromycin selection showed that more than half contained the entire stacked T-DNA (based on phenotype and genotype assays). The other events, based on genomic PCR analyses, were typically lacking portions of the T-DNA which explained their failure to produce the expected phenotypes. Since selection of transgenic plants was conducted using only hygromycin selection, it is not surprising that most of the missing T-DNA regions were located within the middle or RB proximal regions of the GAANTRY T-DNAs. This suggests that in the future, if researchers want to more frequently recover events carrying an entire large stacked construct, localizing selection markers near both the left and right borders of the T-DNA and utilizing two selection agents within the transformation and regeneration process will likely enrich for the recovery of events carrying the entire desired construct. However, even with hygromycin selection only, $19 \%$ of the 11-stack events were high-quality desirable lines that appeared to carry 1 complete copy of the $37.4 \mathrm{~kb}$ T-DNA, were backbone-free and expressed all eight of the detectable introduced phenotypes.

\section{Conclusion}

To date, GAANTRY strains containing multigene TDNA stacks have been successful at producing highquality transgenic events in the model plant Arabidopsis (Collier et al. 2018) the Solanaceous tuber crop of potato (McCue et al. 2019) and now the staple cereal crop of rice. The system has been shown to generate low copy, backbone-free, functional events at a substantial frequency with large T-DNA constructs. In this study, six (38\%) and seven (19\%) 5-stack and 11-stack GAANTRY lines appeared to have one complete T-DNA since they have a single copy of hptII, GUSPlus and nptII (11-stack only) transgenes and expressed all the introduced functional traits. Additionally, out of all 53 transgenic rice events, only a single 5 -stack line tested positive for sequences outside of the T-DNA construct, showing that the GAANTRY system most frequently allows the transfer and integration of only the T-DNA sequences. The results presented show that the GAANTRY system provides a simple and reliable method to generate large TDNA constructs that can be efficiently used to produce high-quality transgenic rice.

\section{Supplementary Information}

The online version contains supplementary material available at https://doi. org/10.1186/s12284-021-00460-5.
Additional file 1: Supplemental Table 1. Primers used for genomic $P C R$ reactions to validate the insertion of cargo sequences within the GAANTRY 5-stack and 11-stack strains and the molecular characterization of transgenic rice events. Related to Figs. 2, 4 and 5.

Additional file 2: Supplemental Table 2. Summary of the phenotype and genotype data for the 5-stack transgenic events. Related to Figs. 4 and 6 .

Additional file 3: Supplemental Table 3. Summary of the phenotype and genotype data for the 11-stack transgenic events. Related to Figs. 3, 5 and 6.

Additional file 4: Supplemental Table 4. Primers and probes used in ddPCR analysis. Related to Fig. 6.

\section{Abbreviations}

attB: attachment Bacteria recognition site; attP: attachment Phage recognition site; bar: bialaphos resistance; CaMV 35S: Cauliflower Mosaic Virus 35S; CDS: Gene coding sequence; DNA: Deoxyribonucleic Acid; ddPCR: Droplet digital Polymerase Chain Reaction; eGFP: Enhanced Green Fluorescent Protein; EPSPS: 5-enol-pyruvylshikimate-3-phospate synthase; Fluc: Firefly luciferase; FAM: 6-fluorescein; GAANTRY: Gene Assembly in Agrobacterium by Nucleic acid Transfer using Recombinase technology; GmR: Gentamicin Resistance; HEX: Hexachloro-fluorescein; GUSPlus: $\beta$-glucuronidase; hptll: Hygromycin phosphotransferase 2, LB: T-DNA Left Border; MRS: Multimer Resolution Site; nptll: Neomycin phosphotransferase 2; nos: Nopaline synthase; OsCc1: Oryza sativa Cytochrome C1; OsLP2: Oryza sativa Leaf Panicle 2; OsPS2: Oryza sativa Pollen Specific 2; OsRoot6: Oryza sativa Root 6; OsUBC: Ubiquitin-Conjugating Enzyme E2; PCR: Polymerase Chain Reaction; pRi: Agrobacterium rhizogenes virulence plasmid; PvUbi1: Panicum virgatum Ubiquitin 1; RB: T-DNA Right Border; Rluc: Renilla luciferase; RUBQ2: Rice Ubiquitin 2; TBS: Transformation Booster Sequence; T-DNA: Transfer-DNA; tdTom: tdTomato endoplasmic reticulum localized red fluorescent protein; X-gluc: 5-bromo-4-chloro-3indolyl-B-D-glucuronic acid; ZmUbir: Zea mays Ubiquitin 1

\section{Acknowledgements}

We wish to acknowledge excellent technical support from Toni Mohr, Hailey Park and Bryan Tarape Hernandez.

\section{Authors' Contributions}

LTH and RT designed the research. LTH and JH performed the research. $L T H$, JGT and RT analyzed the data and wrote the manuscript. The authors read and approved the final manuscript.

\section{Funding}

This research was supported by the USDA Agricultural Research Service CRIS project 2030-21220-002-00-D. Mention of trade names or commercial products is solely for the purpose of providing specific information and does not imply recommendation or endorsement by the U.S. Department of Agriculture. USDA is an equal opportunity provider and employer.

\section{Availability of Data and Materials}

Most of the data generated or analyzed during this study are included in this published article and its supplementary information files. Additional data or materials not included in published article are available from the corresponding author on reasonable request.

Ethics Approval and Consent to Participate Not applicable.

Consent for Publication

Not applicable.

Competing Interests

The authors declare that they have no competing interests. 
Received: 20 October 2020 Accepted: 28 January 2021

Published online: 06 February 2021

\section{References}

Bao J (2018) Biotechnology for rice grain quality improvement. In: Rice: chemistry and technology

Binder A, Lambert J, Morbitzer R et al (2014) A modular plasmid assembly kit for multigene expression, gene silencing and silencing rescue in plants. PLoS One 9:e88218. https://doi.org/10.1371/journal.pone.0088218

Biswal AK, Shamim M, Cruzado K et al (2017) Role of biotechnology in rice production. In: Rice production worldwide

Brar DS, Khush GS (2013) Biotechnological approaches for increasing productivity and sustainability of rice production. In: Agricultural sustainability

Buntru M, Gärtner S, Staib L et al (2013) Delivery of multiple transgenes to plant cells by an improved version of MultiRound gateway technology. Transgenic Res 22:153-167. https://doi.org/10.1007/s1 1248-012-9640-0

Cermak T, Curtin SJ, Gil-Humanes J et al (2017) A multi-purpose toolkit to enable advanced genome engineering in plants. Plant Cell. https://doi.org/10.1105/ tpc. 16.00922

Collier R, Bragg J, Hernandez BT, Vogel JP, Thilmony R (2016) Use of Agrobacterium rhizogenes Strain $18 \mathrm{r} 12 \mathrm{v}$ and Paromomycin Selection for Transformation of Brachypodium distachyon and Brachypodium sylvaticum. Frontiers in Plant Science 7

Collier R, Dasgupta K, Xing Y-P et al (2017) Accurate measurement of transgene copy number in crop plants using droplet digital PCR. Plant J 90:1014-1025. https://doi.org/10.1111/tpj.13517

Collier R, Thomson JG, Thilmony R (2018) A versatile and robust agrobacteriumbased gene stacking system generates high-quality transgenic Arabidopsis plants. Plant J 95:573-583. https://doi.org/10.1111/tpj.13992

Cook M, Thilmony R (2012) The OsGEX2 gene promoter confers sperm cell expression in transgenic Rice. Plant Mol Biol Report 30. https://doi.org/10. 1007/s11105-012-0429-3

Dafny-Yelin M, Tzfira T (2007) Delivery of multiple transgenes to plant cells. Plant Physiol 145:1118-1128. https://doi.org/10.1104/pp.107.106104

de Pater BS, van der Mark F, Rueb S, Katagiri F, Chua NH, Schilperoort RA, Hensgens LA (1992) The promoter of the rice gene GOS2 is active in various different monocot tissues and binds rice nuclear factor ASF-1. Plant J 2(6): $837-844$

Hily J-M, Singer S, Yang Y, Liu Z (2009) A transformation booster sequence (TBS) from Petunia hybrida functions as an enhancer-blocking insulator in Arabidopsis thaliana. Plant Cell Rep 28:1095-1104. https://doi.org/10.1007/ s00299-009-0700-8

Jain M, Nijhawan A, Tyagi AK, Khurana JP (2006) Validation of housekeeping genes as internal control for studying gene expression in rice by quantitative real-time PCR. Biochem Biophys Res Commun 345(2):646-651

Jefferson RA, Kavanagh TA, Bevan MW (1987) GUS fusions: $\beta$-glucuronidase as a sensitive and versatile gene fusion marker in higher plants. EMBO I 6:3901-3907

Keravala A, Groth A, Jarrahian S et al (2006) A diversity of serine phage integrases mediate site-specific recombination in mammalian cells. Mol Gen Genomics 276:135-146. https://doi.org/10.1007/s00438-006-0129-5

Lassner MW, Peterson P, Yoder II (1989) Simultaneous amplification of multiple DNA fragments by polymerase chain reaction in the analysis of transgenic plants and their progeny. Plant Mol Biol Rep 7(2):116-128

Li Z, Moon BP, Xing A et al (2010) Stacking multiple transgenes at a selected genomic site via repeated recombinase mediated DNA cassette exchanges. Plant Physiol 154:622-631. https://doi.org/10.1104/pp.110.160093

Ma L, Dong J, Jin Y et al (2011) RMDAP: a versatile, ready-to-use toolbox for multigene genetic transformation. PLoS One 6:e19883. https://doi.org/10. 1371/journal.pone.0019883

Mann DGJ, King ZR, Liu W et al (2011) Switchgrass (Panicum virgatum L.) polyubiquitin gene (PvUbi1 and PvUbi2) promoters for use in plant transformation. BMC Biotechnol 11:74. https://doi.org/10.1186/1472-6750-11-74

McCue KF, Gardner E, Chan R et al (2019) Transgene stacking in potato using the GAANTRY system. BMC Res Notes 12:457. https://doi.org/10.1186/s13104-0194493-8

Nandy S, Zhao S, Pathak BP et al (2015) Gene stacking in plant cell using recombinases for gene integration and nucleases for marker gene deletion. BMC Biotechnol 15:93. https://doi.org/10.1186/s12896-015-0212-2

Oo M, Bae H-K, Nguyen T et al (2014) Evaluation of rice promoters conferring pollen-specific expression in a heterologous system, Arabidopsis. Plant Reprod 27:47-58. https://doi.org/10.1007/s00497-014-0239-x
Pathak B, Srivastava V (2020) Recombinase-mediated integration of a multigene cassette in rice leads to stable expression and inheritance of the stacked locus. Plant Direct. https://doi.org/10.1002/pld3.236

Shih PM, Vuu K, Mansoori N et al (2016) A robust gene-stacking method utilizing yeast assembly for plant synthetic biology. Nat Commun 7:13215. https://doi. org/10.1038/ncomms 13215

Srivastava V, Thomson J (2016) Gene stacking by recombinases. Plant Biotechnol J 14:471-482. https://doi.org/10.1111/pbi.12459

Thilmony R, Guttman M, Thomson JG, Blechl AE (2009) The LP2 leucine-rich repeat receptor kinase gene promoter directs organ-specific, light-responsive expression in transgenic rice. Plant Biotechnol J 7:867-882

Thomson JG, OW DW (2006) Site-specific recombination systems for the genetic manipulation of eukaryotic genomes. Genesis 44:465-476. https://doi.org/10. 1002/dvg.20237

Thomson JG, Yau Y-Y, Blanvillain R et al (2009) ParA resolvase catalyzes sitespecific excision of DNA from the Arabidopsis genome. Transgenic Res 18: 237-248

Untergasser A, Bijl GJM, Liu W et al (2012) One-step agrobacterium mediated transformation of eight genes essential for rhizobium symbiotic signaling using the novel binary vector system pHUGE. PLoS One 7:e47885. https://doi. org/10.1371/journal.pone.0047885

Weber E, Engler C, Gruetzner R et al (2011) A modular cloning system for standardized assembly of multigene constructs. PLoS One 6:e16765. https:// doi.org/10.1371/journal.pone.0016765

Zeevi V, Liang Z, Arieli U, Tzfira T (2012) Zinc finger nuclease and homing endonuclease-mediated assembly of multigene plant transformation vectors. Plant Physiol 158:132-144. https://doi.org/10.1104/pp.111.184374

Zhang H-Y, Wang X-H, Dong L et al (2017) MISSA 2.0: an updated synthetic biology toolbox for assembly of orthogonal CRISPR/Cas systems. Sci Rep 7: 41993. https://doi.org/10.1038/srep41993

Zhu Q, Yu S, Zeng D et al (2017) Development of "purple endosperm rice" by engineering anthocyanin biosynthesis in the endosperm with a highefficiency transgene stacking system. Mol Plant 10:918-929. https://doi.org/ 10.1016/j.molp.2017.05.008

\section{Publisher's Note}

Springer Nature remains neutral with regard to jurisdictional claims in published maps and institutional affiliations.

\section{Submit your manuscript to a SpringerOpen ${ }^{\circ}$ journal and benefit from:}

- Convenient online submission

- Rigorous peer review

- Open access: articles freely available online

High visibility within the field

- Retaining the copyright to your article

Submit your next manuscript at $\boldsymbol{\sim}$ springeropen.com 